\title{
Five year results of rotator cuff repair
}

\author{
Hans Habernek, Lothar Schmid, Eva Frauenschuh
}

\begin{abstract}
In thirty nine patients with either an acute rotator cuff rupture or a chronic impingement syndrome plus a cuff tear, a standard acromioplasty was performed along with a cuff repair using a bone detaching approach. Postoperative active motion was allowed in all but three. Follow up examination was performed two and five years after the operation. Continuous improvement in function, range of movement, and strength was observed, while pain increased slightly. The size of the tear and delay in treatment were determining factors in the outcome.

(Br F Sports Med 1999;33:430-433)
\end{abstract}

Keywords: shoulder; rotator cuff tear; acromioplasty; osseous deltoid detachment; physiotherapy

Arthroscopic subacromial decompression and even arthroscopic repair of a torn rotator cuff has recently become the treatment of choice in shoulder surgery. ${ }^{1-3}$ However, only a few surgeons have enough patients to become skilled in arthroscopic shoulder surgery. A previously published paper advocated a modified approach, using osseous detachment of the deltoid muscle and transosseous repair through modified Kessler sutures (fig 1), with the occurrence of only minor complications. ${ }^{4}$ Although it may be provoking to encourage an open method nowadays, we present the two and five year results for these patients.

\section{Materials and methods}

PATIENTS

Of 215 patients admitted to our trauma department with impingement problems of the shoulder between 1987 and 1993, open acromioplasty with the modified approach was

Trauma Department, Landeskrankenhaus, A-4820 Bad Ischl, Austria

H Habernek

L Schmid

E Frauenschuh

Correspondence to: Dr H Habernek.

Accepted for publication 29 July 1999
Figure 1 Schematic drawing of the osseous detached deltoid muscle and its repair using modified Kessler sutures.

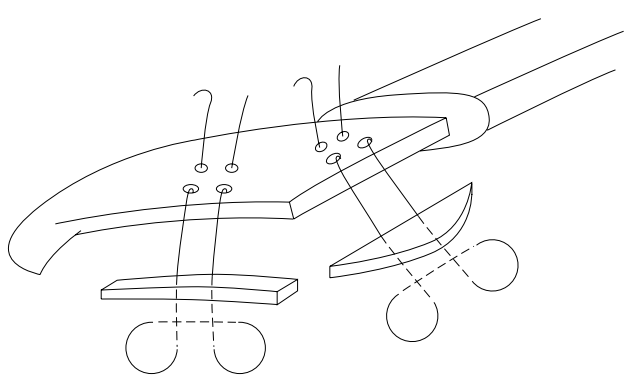

performed in thirty nine (figs 1 and 2). Nineteen of them suffered from an acutely torn rotator cuff, and the remaining 20 had this operation for chronic impingement syndrome (tables 1 and 2). ${ }^{4}$ All were followed up two and five years on average after the operation, and Constant's and Ellman's scores were used. ${ }^{5}{ }^{6}$ The extent of the tear was classified as described by Bateman ${ }^{7}$ and Patte. ${ }^{8}$ After clinical examination including an impingement sign test with bupivacaine, radiographs were taken in an anteroposterior and an axillary view at both follow ups. Preoperative arthrography had been performed in eight patients, while 31 had had ultrasonography before and after treatment. ${ }^{2-11}$ Table 1 presents the patient data. ${ }^{4}$

OPERATION AND POSTOPERATIVE REGIMEN

The procedure was performed as described (figs 1 and 2). ${ }^{4}$ In two cases a polygalactine mesh was used as an interposition because the cuff could not be mobilised sufficiently, but

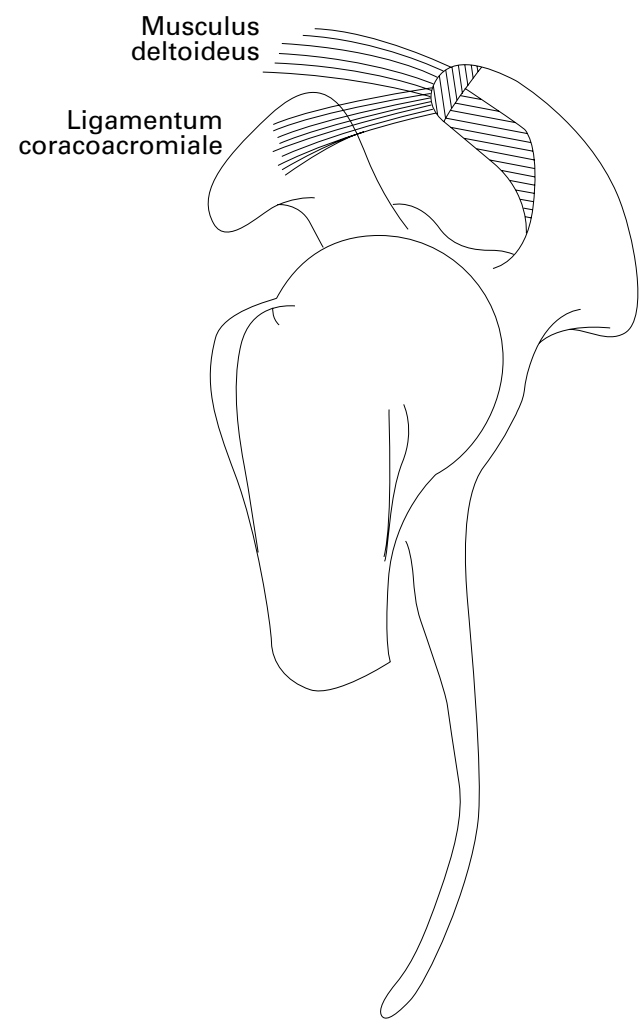

Figure 2 Lateral view of left shoulder joint. The structures to be resected during acromioplasty are hatched. 
Table 1 Two and five year results of surgery for acute rotator cuff rupture $(n=19)$ and chronic impingement syndrome $(n=20)(U C L A \text { score })^{6}$

\begin{tabular}{|c|c|c|c|c|c|c|c|c|c|c|c|c|c|c|c|c|c|c|c|c|c|c|}
\hline \multirow{2}{*}{$\frac{\text { Case }}{1(21)^{\mathrm{a}}}$} & \multirow{2}{*}{$\begin{array}{l}\text { Sex } \\
\mathrm{m}\end{array}$} & \multirow{2}{*}{$\begin{array}{l}\text { Age } \\
48\end{array}$} & \multirow{2}{*}{$\frac{\text { Bateman }}{3}$} & \multirow{2}{*}{$\frac{\text { Patte }^{b}}{3-5-1}$} & \multicolumn{3}{|c|}{ Pain } & \multicolumn{3}{|c|}{ Function } & \multicolumn{3}{|c|}{$R O M$} & \multicolumn{3}{|c|}{ Strength } & \multicolumn{3}{|c|}{ Satisfaction } & \multicolumn{3}{|c|}{ Score } \\
\hline & & & & & 4 & 10 & 10 & 2 & 10 & 10 & 1 & 5 & 5 & 2 & 3 & 5 & 0 & 5 & 5 & 9 & 33 & 35 \\
\hline $2(22)^{a}$ & $\mathrm{~m}$ & 49 & 3 & $3-5-1$ & 4 & 10 & 10 & 2 & 10 & 10 & 1 & 5 & 5 & 2 & 3 & 5 & 0 & 5 & 5 & 9 & 33 & 35 \\
\hline $3(23)^{a}$ & $\mathrm{~m}$ & 37 & 3 & $3-5-1$ & 4 & 10 & 10 & 2 & 10 & 10 & 1 & 5 & 5 & 2 & 3 & 5 & 0 & 5 & 5 & 9 & 33 & 35 \\
\hline $4(24)^{\mathrm{a}}$ & $\mathrm{m}$ & 47 & 3 & $3-5-1$ & 4 & 10 & 10 & 2 & 10 & 10 & 1 & 5 & 5 & 2 & 3 & 5 & 0 & 5 & 5 & 9 & 33 & 35 \\
\hline $5(25)^{\mathrm{a}}$ & $\mathrm{m}$ & 50 & 3 & $3-5-1$ & 4 & 10 & 10 & 2 & 10 & 10 & 1 & 5 & 5 & 2 & 3 & 5 & 0 & 5 & 5 & 9 & 33 & 35 \\
\hline 9 & $\mathrm{~m}$ & 36 & 3 & $3-5-1$ & 4 & 10 & 10 & 2 & 10 & 10 & 1 & 5 & 5 & 2 & 3 & 5 & 0 & 5 & 5 & 9 & 33 & 35 \\
\hline 10 & $\mathrm{f}$ & 48 & 3 & $3-5-1$ & 4 & 10 & 10 & 2 & 10 & 10 & 1 & 5 & 5 & 2 & 3 & 5 & 0 & 5 & 5 & 9 & 33 & 35 \\
\hline 11 & $\mathrm{~m}$ & 49 & 3 & $3-5-2$ & 4 & 10 & 10 & 2 & 10 & 10 & 1 & 5 & 5 & 2 & 3 & 5 & 0 & 5 & 5 & 9 & 33 & 35 \\
\hline 16 & $\mathrm{~m}$ & 54 & 4 & $3-2+5-2$ & 4 & 8 & 10 & 2 & 10 & 10 & 1 & 3 & 4 & 2 & 3 & 5 & 0 & 5 & 5 & 8 & 29 & 34 \\
\hline 15 & $\mathrm{~m}$ & 51 & 4 & $3-6-2$ & 4 & 8 & 10 & 2 & 10 & 10 & 1 & 3 & 4 & 2 & 3 & 4 & 0 & 5 & 5 & 8 & 29 & 33 \\
\hline 12 & $\mathrm{f}$ & 44 & 3 & $3-5-1$ & 4 & 10 & 6 & 2 & 10 & 10 & 1 & 5 & 5 & 2 & 3 & 5 & 0 & 5 & 5 & 9 & 33 & 31 \\
\hline $17^{\text {cd }}$ & $\mathrm{m}$ & 58 & 4 & $3-2+5-2$ & 4 & 8 & 8 & 2 & 10 & 10 & 1 & 3 & 3 & 2 & 3 & 5 & 0 & 5 & 5 & 8 & 29 & 31 \\
\hline 18 & $\mathrm{~m}$ & 43 & 3 & $3-5-1$ & 4 & 10 & 6 & 2 & 10 & 10 & 1 & 5 & 5 & 2 & 3 & 4 & 0 & 5 & 5 & 9 & 33 & 30 \\
\hline 19 & $\mathrm{~m}$ & 49 & 3 & $3-5-1$ & 4 & 10 & 6 & 2 & 10 & 10 & 1 & 5 & 5 & 2 & 3 & 4 & 0 & 5 & 5 & 9 & 33 & 30 \\
\hline $6(26)^{\mathrm{ad}}$ & $\mathrm{m}$ & 49 & 4 & $3-2+5-3$ & 4 & 8 & 6 & 2 & 10 & 10 & 1 & 3 & 4 & 2 & 3 & 5 & 0 & 5 & 5 & 8 & 29 & 30 \\
\hline $7(27)^{\text {acd }}$ & $\mathrm{m}$ & 45 & 4 & $3-2+5-3$ & 4 & 8 & 6 & 2 & 10 & 10 & 1 & 3 & 4 & 2 & 3 & 5 & 0 & 5 & 5 & 8 & 29 & 30 \\
\hline 13 & $\mathrm{f}$ & 47 & 3 & $3-5-2$ & 4 & 10 & 6 & 2 & 10 & 10 & 1 & 4 & 4 & 2 & 3 & 4 & 0 & 5 & 5 & 9 & 32 & 29 \\
\hline $14^{\mathrm{d}}$ & $\mathrm{m}$ & 43 & 3 & $3-5-1$ & 4 & 10 & 6 & 2 & 10 & 10 & 1 & 5 & 5 & 2 & 3 & 4 & 0 & 5 & 5 & 9 & 33 & 25 \\
\hline $8(28)^{\text {acd }}$ & $\mathrm{m}$ & 63 & 4 & $3-2+5-3$ & 2 & 8 & 6 & 1 & 4 & 6 & 0 & 2 & 3 & 2 & 3 & 4 & 0 & 0 & 0 & 5 & 17 & 19 \\
\hline 1 & $\mathrm{~m}$ & 45 & 2 & $3-3-1$ & 2 & 10 & 8 & 4 & 10 & 10 & 2 & 5 & 5 & 4 & 5 & 5 & 0 & 5 & 5 & 12 & 35 & 33 \\
\hline 2 & $\mathrm{~m}$ & 49 & 2 & $3-3-1$ & 2 & 10 & 8 & 4 & 10 & 10 & 2 & 5 & 5 & 4 & 5 & 5 & 0 & 5 & 5 & 12 & 35 & 33 \\
\hline 3 & $\mathrm{~m}$ & 69 & 2 & $3-3-1$ & 2 & 10 & 8 & 4 & 10 & 10 & 2 & 5 & 5 & 4 & 5 & 5 & 0 & 5 & 5 & 12 & 35 & 33 \\
\hline 4 & $\mathrm{f}$ & 49 & 2 & $3-3-1$ & 2 & 10 & 8 & 4 & 10 & 10 & 2 & 5 & 5 & 4 & 5 & 5 & 0 & 5 & 5 & 12 & 35 & 33 \\
\hline 5 & $\mathrm{~m}$ & 57 & 2 & $3-3-1$ & 2 & 10 & 8 & 4 & 10 & 10 & 2 & 5 & 5 & 4 & 5 & 5 & 0 & 5 & 5 & 12 & 35 & 33 \\
\hline 6 & $\mathrm{~m}$ & 39 & 2 & $3-3-1$ & 2 & 10 & 8 & 4 & 10 & 10 & 2 & 5 & 5 & 4 & 5 & 5 & 0 & 5 & 5 & 12 & 35 & 33 \\
\hline 7 & $\mathrm{~m}$ & 30 & 2 & $3-3-1$ & 2 & 10 & 8 & 4 & 10 & 10 & 2 & 5 & 5 & 4 & 5 & 5 & 0 & 5 & 5 & 12 & 35 & 33 \\
\hline 8 & f & 61 & 2 & $3-3-1$ & 2 & 10 & 8 & 4 & 10 & 10 & 2 & 5 & 5 & 4 & 5 & 5 & 0 & 5 & 5 & 12 & 35 & 33 \\
\hline 9 & $\mathrm{f}$ & 37 & 2 & $3-3-1$ & 2 & 8 & 8 & 4 & 10 & 10 & 2 & 5 & 5 & 4 & 5 & 5 & 0 & 5 & 5 & 12 & 35 & 33 \\
\hline 10 & $\mathrm{~m}$ & 40 & 2 & $3-3-1$ & 2 & 8 & 8 & 4 & 10 & 10 & 2 & 5 & 5 & 4 & 5 & 5 & 0 & 5 & 5 & 12 & 35 & 33 \\
\hline 11 & f & 49 & 2 & $3-3-1$ & 2 & 8 & 8 & 4 & 10 & 10 & 2 & 5 & 5 & 4 & 5 & 5 & 0 & 5 & 5 & 12 & 35 & 33 \\
\hline 12 & $\mathrm{~m}$ & 47 & 2 & $3-3-1$ & 2 & 8 & 8 & 4 & 10 & 10 & 2 & 5 & 5 & 4 & 5 & 5 & 0 & 5 & 5 & 12 & 35 & 33 \\
\hline 13 & $\mathrm{~m}$ & 48 & 2 & $3-4-1$ & 2 & 8 & 8 & 4 & 10 & 10 & 2 & 5 & 5 & 4 & 5 & 5 & 0 & 5 & 5 & 12 & 35 & 33 \\
\hline 14 & $\mathrm{~m}$ & 43 & 2 & $3-4-1$ & 2 & 10 & 8 & 4 & 10 & 10 & 2 & 5 & 5 & 4 & 5 & 5 & 0 & 5 & 5 & 12 & 35 & 33 \\
\hline 15 & $\mathrm{~m}$ & 51 & 2 & $3-4-1$ & 2 & 10 & 8 & 4 & 10 & 10 & 2 & 5 & 5 & 4 & 5 & 5 & 0 & 5 & 5 & 12 & 35 & 33 \\
\hline 16 & $\mathrm{~m}$ & 44 & 2 & $3-5-1$ & 2 & 10 & 8 & 4 & 10 & 10 & 2 & 5 & 5 & 4 & 5 & 5 & 0 & 5 & 5 & 12 & 35 & 33 \\
\hline 17 & $\mathrm{~m}$ & 39 & 3 & $3-5-1$ & 2 & 10 & 8 & 4 & 10 & 10 & 2 & 5 & 5 & 4 & 5 & 5 & 0 & 5 & 5 & 12 & 35 & 33 \\
\hline 18 & $\mathrm{~m}$ & 48 & 3 & $3-5-1$ & 2 & 10 & 8 & 4 & 10 & 10 & 2 & 5 & 5 & 4 & 5 & 5 & 0 & 5 & 5 & 12 & 35 & 33 \\
\hline 19 & $\mathrm{~m}$ & 56 & 3 & $3-5-1$ & 2 & 8 & 8 & 4 & 10 & 10 & 2 & 5 & 5 & 4 & 5 & 5 & 0 & 5 & 5 & 12 & 35 & 33 \\
\hline 20 & $\mathrm{~m}$ & 53 & 3 & $3-5-1$ & 2 & 8 & 8 & 4 & 10 & 10 & 2 & 5 & 5 & 4 & 5 & 5 & 0 & 5 & 5 & 12 & 35 & 33 \\
\hline
\end{tabular}

${ }^{a}$ Numbers in parentheses are cases published in Acta Orthop Scand 1993;64:87-91.

${ }^{b}$ Patte classification: group-segment-stage.

${ }^{\mathrm{c}}$ Concomitant shoulder joint dislocation.

${ }^{\mathrm{d}}$ Delayed diagnosis.

supraspinatus muscle advancement had not been carried out ${ }^{1213}$ (cases no 7(27) and $8(28)) .{ }^{14}$ All patients showed macroscopic signs of degeneration of the cuff but no histological examination was undertaken.

After ten days in a mitella, active motion up to $90^{\circ}$ was allowed in all but three who received abduction splinting for six weeks (cases no $7(27), 8(28)$, and 15). Sporting activity was begun three months after surgery, starting with swimming.

Results (tables 1 and 2) ${ }^{56}$

ACUTE CUFF RUPTURES

With increasing extent of the tear, pain was increased and range of movement (ROM) and strength were decreased. Two poor and four moderate results contrasted with 13 good results. Although function had remained the same, there was deterioration with regard to pain. None had changed employment, and sports were practised by all but one. Ultrasound examination showed no tear at this date except in the two patients with a poor outcome. Sick leave averaged ten weeks.

IMPINGEMENT SYNDROMES

After two years, function, ROM, and strength had returned to normal and all were satisfied. Pain had not improved in 10 patients. Similar results were seen after five years except for occasional pain in all patients. Sick leave aver- aged ten weeks but white collar workers had four weeks on average.

\section{COMPLICATIONS (TABLE 3)}

No infection, axillary or suprascapular nerve injury, unrecognised unstable os acromiale lesion, or failed subacromial decompression were found at follow up.

\section{Discussion}

The main focus of this paper was to propose an open approach and a cuff repair in patients suffering from either an acute major cuff rupture or an impingement problem associated with a major cuff rupture (a full thickness tear of 1-3 cm or more (Bateman 3) involving one or more tendons), who wish to participate further in overhead sports. The bone detaching approach was used because of the clear view obtained and its low morbidity, whereas subperiosteal detachment was not found to be reliable for early active motion after repair of massive tears. ${ }^{4} 9^{15-17}$

If the tear did not involve "more than the supraspinatus and (part of) the infraspinatus tendon, possessed edge stability and occurred through a minimal surface area", conservative treatment was also feasible. ${ }^{18-20}$ Massive cuff tears $(>5 \mathrm{~cm}=$ Bateman IV) in which the torn tendons involve the supraspinatus muscle alone may be treated by endoscopic subacromial decompression and rotator cuff debride- 
Table 2 Two and five year results of surgery for acute rotator cuff rupture $(n=19)$ and chronic impingement syndrome $(n=20)(\text { Constant score })^{5}$

\begin{tabular}{|c|c|c|c|c|c|c|c|c|c|c|c|c|c|c|c|c|c|c|c|c|c|c|c|}
\hline \multirow{2}{*}{$\frac{\text { Case }}{1(21)^{\mathrm{a}}}$} & \multirow{2}{*}{$\frac{\operatorname{Sex}}{\mathrm{m}}$} & \multirow{2}{*}{$\begin{array}{l}\text { Age } \\
48\end{array}$} & \multirow{2}{*}{$\begin{array}{l}\text { Bateman } \\
3\end{array}$} & \multirow{2}{*}{$\frac{\text { Patte }^{b}}{3-5-1}$} & \multicolumn{3}{|l|}{ Pain } & \multicolumn{3}{|c|}{ Activity level } & \multicolumn{3}{|c|}{ Positioning } & \multicolumn{3}{|c|}{$R O M$} & \multicolumn{3}{|c|}{ Strength } & \multicolumn{4}{|c|}{ Score } \\
\hline & & & & & 0 & 15 & 15 & 0 & 10 & 10 & 4 & 10 & 10 & 6 & 40 & 40 & 0 & 20 & 20 & 10 & 95 & 95 & Excellent \\
\hline $2(22)^{a}$ & $\mathrm{~m}$ & 49 & 3 & $3-5-1$ & 0 & 15 & 15 & 0 & 10 & 10 & 4 & 10 & 10 & 6 & 40 & 40 & 0 & 20 & 20 & 10 & 95 & 95 & \\
\hline $3(23)^{\mathrm{a}}$ & $\mathrm{m}$ & 37 & 3 & $3-5-1$ & 0 & 15 & 15 & 0 & 10 & 10 & 4 & 10 & 10 & 6 & 40 & 40 & 0 & 20 & 20 & 10 & 95 & 95 & \\
\hline $4(24)^{\mathrm{a}}$ & $\mathrm{m}$ & 47 & 3 & $3-5-1$ & 0 & 15 & 15 & 0 & 10 & 10 & 4 & 10 & 10 & 8 & 40 & 40 & 0 & 20 & 20 & 12 & 95 & 95 & \\
\hline $5(25)^{a}$ & $\mathrm{~m}$ & 50 & 3 & $3-5-1$ & 0 & 15 & 15 & 0 & 10 & 10 & 4 & 10 & 10 & 8 & 40 & 40 & 0 & 20 & 20 & 12 & 95 & 95 & \\
\hline 9 & $\mathrm{~m}$ & 36 & 3 & $3-5-1$ & 0 & 15 & 15 & 0 & 10 & 10 & 4 & 10 & 10 & 8 & 40 & 40 & 0 & 20 & 20 & 12 & 95 & 95 & \\
\hline 10 & f & 48 & 3 & $3-5-1$ & 0 & 15 & 15 & 0 & 10 & 10 & 4 & 10 & 10 & 10 & 40 & 40 & 0 & 20 & 20 & 14 & 95 & 95 & \\
\hline 15 & $\mathrm{~m}$ & 49 & 3 & $3-5-2$ & 0 & 15 & 15 & 0 & 10 & 10 & 4 & 10 & 10 & 12 & 36 & 36 & 0 & 20 & 20 & 16 & 91 & 91 & \\
\hline 11 & $\mathrm{~m}$ & 54 & 4 & $3-2+5-2$ & 0 & 10 & 10 & 0 & 10 & 10 & 4 & 10 & 10 & 10 & 40 & 40 & 0 & 20 & 20 & 14 & 90 & 90 & \\
\hline 16 & $\mathrm{~m}$ & 51 & 4 & $3-6-2$ & 0 & 15 & 15 & 0 & 10 & 10 & 4 & 10 & 10 & 10 & 32 & 34 & 5 & 20 & 20 & 19 & 87 & 89 & \\
\hline $17^{\text {cd }}$ & $\mathrm{f}$ & 44 & 3 & $3-5-1$ & 0 & 5 & 10 & 0 & 10 & 10 & 4 & 10 & 10 & 8 & 38 & 38 & 0 & 20 & 20 & 12 & 83 & 88 & \\
\hline 12 & $\mathrm{~m}$ & 58 & 4 & $3-2+5-2$ & 0 & 5 & 10 & 0 & 10 & 10 & 4 & 10 & 10 & 12 & 30 & 32 & 5 & 20 & 20 & 21 & 75 & 82 & \\
\hline 18 & $\mathrm{~m}$ & 43 & 3 & $3-5-1$ & 0 & 5 & 5 & 0 & 8 & 8 & 4 & 10 & 10 & 8 & 38 & 32 & 0 & 20 & 20 & 12 & 81 & 75 & \\
\hline 19 & $\mathrm{~m}$ & 49 & 3 & $3-5-1$ & 0 & 5 & 10 & 0 & 8 & 8 & 4 & 10 & 10 & 10 & 34 & 26 & 5 & 20 & 20 & 19 & 77 & 74 & \\
\hline $6(26)^{\mathrm{ad}}$ & $\mathrm{m}$ & 49 & 4 & $3-2+5-3$ & 0 & 10 & 10 & 0 & 6 & 6 & 4 & 10 & 8 & 6 & 32 & 28 & 5 & 20 & 20 & 15 & 78 & 72 & \\
\hline $7(27)^{\text {acd }}$ & $\mathrm{m}$ & 45 & 4 & $3-2+5-3$ & 0 & 10 & 10 & 0 & 6 & 6 & 4 & 10 & 8 & 6 & 32 & 28 & 0 & 20 & 20 & 10 & 78 & 72 & \\
\hline 13 & $\mathrm{f}$ & 47 & 3 & $3-5-2$ & 0 & 5 & 5 & 0 & 10 & 10 & 4 & 10 & 10 & 12 & 38 & 34 & 0 & 10 & 10 & 16 & 73 & 69 & \\
\hline $14^{\text {ad }}$ & $\mathrm{m}$ & 43 & 3 & $3-5-1$ & 0 & 10 & 10 & 0 & 6 & 6 & 4 & 6 & 10 & 4 & 18 & 16 & 0 & 10 & 10 & 8 & 50 & 52 & $\downarrow$ \\
\hline $8(28)^{\text {acd }}$ & $\mathrm{m}$ & 63 & 4 & $3-2+5-3$ & 0 & 10 & 10 & 0 & 6 & 6 & 4 & 6 & 6 & 2 & 12 & 12 & 0 & 5 & 5 & 6 & 39 & 39 & Worse \\
\hline 4 & $\mathrm{~m}$ & 45 & 2 & $3-3-1$ & 10 & 10 & 10 & 4 & 10 & 8 & 6 & 10 & 10 & 12 & 40 & 32 & 0 & 20 & 20 & 32 & 90 & 80 & Excellent \\
\hline 3 & $\mathrm{~m}$ & 49 & 2 & $3-3-1$ & 10 & 10 & 10 & 4 & 10 & 8 & 6 & 10 & 10 & 12 & 40 & 30 & 0 & 20 & 20 & 32 & 90 & 78 & \\
\hline 1 & $\mathrm{~m}$ & 69 & 2 & $3-3-1$ & 10 & 15 & 10 & 4 & 10 & 8 & 6 & 10 & 10 & 12 & 40 & 30 & 0 & 20 & 20 & 32 & 95 & 78 & \\
\hline 2 & f & 49 & 2 & $3-3-1$ & 10 & 15 & 10 & 4 & 10 & 8 & 6 & 10 & 10 & 12 & 40 & 30 & 0 & 20 & 20 & 32 & 95 & 78 & \\
\hline 17 & $\mathrm{~m}$ & 57 & 2 & $3-3-1$ & 10 & 15 & 10 & 4 & 10 & 8 & 6 & 10 & 10 & 12 & 38 & 30 & 0 & 20 & 20 & 32 & 93 & 78 & \\
\hline 5 & $\mathrm{~m}$ & 39 & 2 & $3-3-1$ & 10 & 10 & 10 & 4 & 10 & 8 & 6 & 10 & 8 & 12 & 40 & 30 & 0 & 20 & 20 & 32 & 90 & 76 & \\
\hline 6 & $\mathrm{~m}$ & 30 & 2 & $3-3-1$ & 10 & 10 & 10 & 4 & 10 & 8 & 6 & 10 & 8 & 12 & 40 & 30 & 0 & 20 & 20 & 32 & 90 & 76 & \\
\hline 8 & $\mathrm{f}$ & 61 & 2 & $3-3-1$ & 10 & 10 & 10 & 4 & 10 & 8 & 6 & 10 & 8 & 12 & 40 & 30 & 0 & 20 & 20 & 32 & 90 & 76 & \\
\hline 13 & f & 37 & 2 & $3-3-1$ & 10 & 10 & 10 & 4 & 10 & 8 & 6 & 10 & 10 & 12 & 38 & 28 & 0 & 20 & 20 & 32 & 88 & 76 & \\
\hline 15 & $\mathrm{~m}$ & 40 & 2 & $3-3-1$ & 10 & 10 & 10 & 4 & 8 & 8 & 6 & 10 & 10 & 12 & 38 & 28 & 0 & 20 & 20 & 32 & 86 & 76 & \\
\hline 7 & f & 49 & 2 & $3-3-1$ & 10 & 10 & 10 & 4 & 10 & 8 & 6 & 10 & 8 & 12 & 40 & 28 & 0 & 20 & 20 & 32 & 90 & 74 & \\
\hline 16 & $\mathrm{~m}$ & 47 & 2 & $3-3-1$ & 10 & 15 & 10 & 4 & 10 & 8 & 6 & 10 & 8 & 12 & 38 & 28 & 0 & 20 & 20 & 32 & 93 & 74 & \\
\hline 18 & $\mathrm{~m}$ & 48 & 2 & $3-4-1$ & 10 & 15 & 10 & 4 & 10 & 8 & 6 & 10 & 8 & 12 & 36 & 28 & 0 & 20 & 20 & 32 & 91 & 74 & \\
\hline 14 & $\mathrm{~m}$ & 43 & 2 & $3-4-1$ & 10 & 15 & 10 & 4 & 8 & 8 & 6 & 10 & 10 & 12 & 38 & 22 & 0 & 20 & 20 & 32 & 91 & 70 & \\
\hline 12 & $\mathrm{~m}$ & 51 & 2 & $3-4-1$ & 10 & 15 & 10 & 0 & 10 & 8 & 6 & 10 & 10 & 8 & 32 & 30 & 0 & 10 & 10 & 24 & 77 & 68 & \\
\hline 9 & $\mathrm{~m}$ & 44 & 2 & $3-5-1$ & 10 & 15 & 10 & 0 & 10 & 8 & 6 & 10 & 8 & 8 & 32 & 24 & 0 & 10 & 10 & 24 & 77 & 60 & \\
\hline 19 & $\mathrm{~m}$ & 39 & 3 & $3-5-1$ & 10 & 10 & 10 & 0 & 10 & 8 & 6 & 10 & 10 & 8 & 32 & 20 & 0 & 10 & 10 & 24 & 72 & 58 & \\
\hline 10 & $\mathrm{~m}$ & 48 & 3 & $3-5-1$ & 10 & 15 & 10 & 0 & 10 & 8 & 6 & 10 & 8 & 8 & 32 & 20 & 0 & 10 & 10 & 24 & 77 & 56 & \\
\hline 11 & $\mathrm{~m}$ & 56 & 3 & $3-5-1$ & 10 & 15 & 10 & 0 & 10 & 8 & 6 & 10 & 8 & 8 & 32 & 20 & 0 & 10 & 10 & 24 & 77 & 56 & $\downarrow$ \\
\hline 20 & $\mathrm{~m}$ & 53 & 3 & $3-5-1$ & 10 & 10 & 10 & 0 & 10 & 8 & 6 & 10 & 8 & 8 & 32 & 14 & 0 & 10 & 10 & 24 & 72 & 50 & Worse \\
\hline
\end{tabular}

${ }^{a}$ Numbers in parentheses are cases published in Acta Orthop Scand 1993;64:87-91.

${ }^{b}$ Patte classification: group-segment-stage. ${ }^{8}$

${ }^{\mathrm{C}}$ Concomitant shoulder joint dislocation.

${ }^{\mathrm{d} D e l a y e d}$ diagnosis.

ROM, range of motion.

ment. ${ }^{17}{ }^{21}$ Arthroscopic cuff repair may not be feasible for tears that involve more than the supraspinatus tendon (C Gerber, personal communication). Comparing arthroscopic with open repair was considered to be meaningless as both are indispensable modes of treatment.

Acute rotator cuff tears with functional deficits are rare $(4 \% ; 19$ of 215). Cuff tears concomitant with a shoulder dislocation are reported to occur more often than they are diagnosed. ${ }^{22}$ Being a non-invasive diagnostic procedure, post-reduction sonography should be invaluable in cases of shoulder dislocation. ${ }^{22}$

As magnetic resonance imaging was not available, computed tomographic arthrography was performed in patients with fresh dislocations after ten days. ${ }^{22}$ Although pure labral tears may be treated arthroscopically, the extent of a cuff rupture can be diagnosed arthroscopically, but repaired by open surgery if necessary. Overall, open repair was performed if functional recovery and strength were the primary objectives for patients under the age of 60 who wished to continue with overhead sports activities. ${ }^{1}$ A poor outcome can be expected if a patient with a neglected extensive cuff tear has a long standing functional deficit. A correlation between duration of

Table 3 Complications of surgery $(n=17)$

\begin{tabular}{lclllll}
\hline Complication & Case & $\begin{array}{l}\text { Patte- } \\
\text { Bateman }\end{array}$ & $\begin{array}{l}\text { Time after } \\
\text { surgery }\end{array}$ & Cause & Procedure & Result \\
\hline Deltoid avulsion & $6(26)$ & $3-2+5-3$ & 3 weeks & Sudden dynamic loading & Reoperation & Moderate \\
Impingement & $6(26)$ & $3-2+5-3$ & 5 years & Inadequate resection & Conservatively & Moderate \\
& $7(27)$ & $3-2+5-3$ & 5 years & Inadequate resection & Conservatively & Moderate \\
& $8(28)$ & $3-2+5-3$ & 5 years & Inadequate resecion & Conservatively & Worse \\
& 12 & $3-5-1$ & 5 years & Inadequate resection & Conservatively & Moderate \\
& 14 & $3-5-1$ & 5 years & Inadequate resection & Conservatively & Worse \\
& 18 & $3-5-1$ & 5 years & Inadequate resection & Conservatively & Good \\
& 19 & $3-5-1$ & 5 years & Inadequate resection & Conservatively & Good \\
Non-union & $4(24)$ & $3-5-1$ & 5 years & Inadequate reattachment & Conservatively & Excellent \\
& $5(25)$ & $3-5-1$ & 5 years & Inadequate reattachment & Conservatively & Excellent \\
& 14 & $3-5-1$ & 5 years & Inadequate reattachment & Conservatively & Worse \\
& 17 & $3-2+5-2$ & 5 years & Inadequate reattachment & Conservatively & Moderate \\
& 18 & $3-5-1$ & 5 years & Inadequate reattachment & Conservatively & Good \\
& 20 & $3-5-1$ & 5 years & Inadequate reattachment & Conservatively & Moderate \\
& $6(26)$ & $3-2+5-3$ & 3 weeks & Sudden dynamic loading & Reoperation & Moderate \\
Cuff retearing & $7(27)$ & $3-2+5-3$ & 5 years & Inadequate resection & Conservatively & Moderate \\
& $8(28)$ & $3-2+5-3$ & 5 years & Inadequate resecion & Conservatively & Worse \\
\hline
\end{tabular}


Table 4 Results of surgery for massive rotator cuff tears ${ }^{23}$

\begin{tabular}{llllll}
\hline Authors & $\begin{array}{l}\text { No of } \\
\text { patients }\end{array}$ & $\begin{array}{l}\text { Minimum } \\
\text { follow up } \\
\text { (months) }\end{array}$ & $\begin{array}{l}\text { Overall } \\
\text { results (\%) }\end{array}$ & $\begin{array}{l}\text { Pain } \\
\text { relief }\end{array}$ & $\begin{array}{l}\text { Average active } \\
\text { forward elevation } \\
\text { (degrees) }\end{array}$ \\
\hline Bigliani et al & 61 & 24 & 85 & 95 & 164 \\
Rockwood \& Burkhead & 15 & 60 & - & 100 & 155 \\
Nasca & 7 & 30 & 29 & 71 & 97 \\
Björkenheim et al & 10 & 24 & 30 & - & - \\
Hawkins et al & 27 & 24 & - & 85 & 134 \\
Ozaki et al & 27 & 5 & 93 & - & - \\
Bayne \& Bateman & 30 & 30 & 73 & - & - \\
Ha'eri \& Wiley & 18 & 24 & 78 & 81 & 123 \\
Neviaser et al & 16 & 9 & 88 & 52 & 162 \\
Own results & 19 & 60 & 68 & &
\end{tabular}

${ }^{a}$ Only excellent/good results are presented.

$\mathrm{NB}$ All references can be found in Ianotti. ${ }^{23}$

symptoms, the size of the tear, and the end result has been found.

In the group with chronic impingement syndrome, all presented with a clear cut improvement in function but with slight pain at follow up. For chronic impingement syndromes, open acromioplasty and cuff repair can be delayed until after a physiotherapy regimen of six months has failed and pain and decreased function or diminished ROM are present. The results presented here compare favourably with those of others in spite of different evaluation methods and different techniques (table 4$).^{23}$

1 Burkhart SS. Arthroscopic treatment of massive rotator cuff tears. Clin Orthop 1991;267:45-56.

2 Liu SH. Arthroscopically-assisted rotator-cuff repair. $\mathcal{F}$ Bone foint Surg [Br] 1994;76:592-5.

3 Van Holsbeeck E, DeRyke J, Declerqu G, et al. Subacromial impingement: open versus arthroscopic decompression. Iournal of Arthroscopy 1992;8:173-8.

4 Haurnal of Arthroscopy 1992;8:173-8. to the subacromial space. Acta Orthop Scand 1993;64:92-4. to the subacromial space. Acta Orthop Scand 1993;64:92-4.
Constant CR, Murley AHG. A Clinical method of functional assessment of the shoulder. Clin Orthop $1987 ; 214: 160-4$.
6 Ellman H, Hanker G, Bayer M. Repair of the rotator cuff. End-result study of factors influencing reconstruction. $\mathcal{f}$ End-result study of factors influencing
Bone foint Surg [Am] 1986;68:1136-44.

7 Bateman JE. The diagnosis and treatment of tears of the rotator cuff. Surg Clin North Am 1963;43:1523-34.

8 Patte D. Classification of rotator cuff lesions. Clin Orthop 1990;254:81-6.

9 Neer II CS. Impingement lesions. Clin Orthop 1983;173:707.

10 Andrews JR, Byrd JW, Kupferman SP, et al. The profile view of the acromion. Clin Orthop 1991;263:142-6.

11 Drakeford MK, Quinn MJ, Simpson SL, et al. A comparative study of ultrasonography and arthrography in evaluation of the rotator cuff. Clin Orthop 1990;253:11822

12 Debeyre J, Patte D, Elmelik E. Repair of ruptures of the rotator cuff of the shoulder. F Bone Foint Surg [Br] 1965;47: $36-42$.

$13 \mathrm{Ha}^{\prime}$ eri BG, Wiley AM. Advancement of the supraspinatus muscle in the repair of ruptures of the rotator cuff. J Bone Joint Surg [Am] 1981;63:232-8.

14 Ozaki J, Fujimoto S, Masuhara K, et al. Reconstruction of chronic massive rotator cuff tears with synthetic materials. Clin Orthop 1986;202:173-83.

15 Ogilvie-Harris DJ, Wiley AM, Sattarian J. Failed acromioplasty for impingement syndrome. F Bone foint Surg $[\mathrm{Br}]$ 1990;72:1070-2.

16 Post M. Complications of rotator cuff surgery. Clin Orthop 1990;254:97-104

17 Rockwood CA Jr, Lyons FR. Shoulder impingement syndrome: diagnosis, radiographic evaluation, and treatment with a modified neer acromioplasty. F Bone foint Surg [Am] 1993;75A:409-24.

18 Burkhart SS, Esch JC, Jolson RS. The rotator crescent and rotator cable: an anatomic description of the shoulder's "suspension bridge". Fournal of Arthroscopy 1993;9:61116.

19 Burkhart SS. Reconciling the paradox of rotator cuff repair versus debridement: a unified biomechanical rationale for the treatment of rotator cuff tears. Fournal of Arthroscopy 1994;10:4-19.

20 Itoi E, Tabata S. Conservative treatment of rotator cuff tears. Clin Orthop 1992;275:165-73.

21 Ellman H, Kay SP, Wirth M. Arthroscopic treatment of full-thickness rotator cuff tears: 2- to 7-year follow-up study. Fournal of Arthroscopy 1993;9:195-200.

22 Neviaser RJ, Neviaser TJ, Neviaser JS. Concurrent rupture of the rotator cuff and anterior dislocation of the shoulder in the older patient. F Bone foint Surg [Am] 1988;70:130811 .

23 Ianotti JP. Rotator cuff disorders. Evaluation and treatment. In: Kaufer H, ed. American Academy of Orthopedic Surgeons monograph series. Park Ridge, IL: American Academy of Orthopedic Surgeons, 1991.

Take home message

In the case of a massive rupture of the rotator cuff, acromioplasty and repair of the cuff can effectively complement endoscopic surgery. The osseous deltoid detaching approach allows shoulder mobilisation as early as 10 days after the operation. 